\title{
IMPACTO DA OBESIDADE NA MORTALIDADE DE IDOSOS COM COVID-19
}

Kimberly Mayara Gouveia Bezerra; Universidade Federal de Pernambuco - UFPE; kimberly.gouveia@ufpe.br

Crislayne Maria Berto; Universidade Federal de Pernambuco - UFPE; crislayne.berto@ufpe.br

Fernando Arthur Alves da Silva; Universidade Federal de Pernambuco - UFPE; fernando.arthur@ufpe.br

Leiliane Moraes dos Santos Silva; Universidade Federal de Pernambuco - UFPE; leiliane.moraes@ufpe.br

Ana Paula de Oliveira Marques; Universidade Federal de Pernambuco - UFPE; ana.marques@ufpe.br

\section{RESUM0}

Introdução: A obesidade é um problema de saúde pública e sua prevalência corresponde a 17,9\% na faixa etária de 60 a 74 anos e 15,8\% para os com 75 anos ou mais. Pessoas com obesidade são mais propensas a infecções, pois esta condição prejudica a imunidade ao causar uma inflamação crônica do tecido adiposo, influenciando a atividade das células da imunidade inata e adaptativa, além de perturbar o equilíbrio hormonal do organismo. Além disso, devido ao aumento de tecido adiposo, há alta expressão de receptores ACE2, responsáveis pela entrada do vírus SARS-Cov-2 nas células. Métodos: Realizou-se uma revisão integrativa nas bases de dados Literatura Internacional em Ciências da Saúde (MEDLINE), Scientific Electronic Library Online (SciELO), Centro Latino-Americano e do Caribe de Informação em Ciências da Saúde (LILACS) e Pubmed, utilizando a combinação dos descritores "obesity", "elderly" e "COVID-19". 161 artigos foram encontrados no total, dos quais 23 foram pré-selecionados e, após aplicação dos critérios de elegibilidade, 11 artigos foram incluídos. Resultados: Evidenciou-se que a obesidade é um fator de risco forte e independente para maior gravidade da COVID-19 em idosos, bem como para maior necessidade de suporte respiratório avançado, diminuição da eliminação do vírus e para mortalidade. Níveis mais elevados de índice de massa corporal (IMC) têm relação diretamente proporcional a piores desfechos da doença. Conclusão: A obesidade é um fator de risco para mortalidade em idosos com COVID-19, portanto uma atenção especial deve ser dada na prevenção e controle dessa comorbidade e suas complicações para proteção desta população.

Palavras-chave: Obesidade; COVID-19; Mortalidade; Idoso. 